\title{
ON A CONSEQUENCE OF THE ORDER IDEAL CONJECTURE
}

\author{
S. P. DutTA \\ Dedicated to H.-B. Foxby on his 65th birthday.
}

\begin{abstract}
Given a minimal set of generators $\mathbf{x}$ of an ideal $I$ of height $\mathrm{d}$ in a regular local ring $(R, m, k)$ we prove several cases for which the map $K_{d}(\mathbf{x} ; R) \otimes k \rightarrow \operatorname{Tor}_{d}^{R}(R / I, k)$ is the 0-map. As a consequence of the order ideal conjecture we derive several cases for which $K_{d+i}(\mathbf{x} ; R) \otimes k \rightarrow \operatorname{Tor}_{d+i}^{R}(R / I, k)$ are 0-maps for $i \geq 0$.
\end{abstract}

In order to solve the syzygy problem in the equicharacteristic case Evans and Griffith proved the following:

Theorem A ([E-G1]; Th. 2.4, [E-G2]). Let $R$ be a local ring containing a field. Let $M$ be a finitely generated $k$-th syzygy of finite projective dimension and let $x$ be a minimal generator of $M$. Then the order ideal $\mathcal{O}_{M}(x)=\left\{f(x) \mid f \in \operatorname{Hom}_{R}(M, R)\right\}$ has grade at least $k$.

We drop $M$ from the notation for order ideals when there is no scope for confusion. Afterwards Bruns and Herzog extended the above theorem for finite complexes of finitely generated free modules in the following way.

Theorem B (Th. 9.5.2, $[\mathrm{B}-\mathrm{H}])$. Let $(R, m)$ be a local ring containing a field and let

$$
F_{\bullet}: 0 \rightarrow F_{s} \stackrel{\phi_{s}}{\longrightarrow} F_{s-1} \rightarrow \cdots \rightarrow F_{1} \stackrel{\phi_{1}}{\longrightarrow} F_{0} \rightarrow 0
$$

be a complex of finitely generated free $R$-modules. Then for every $j, 1 \leq j \leq s$ and for every $e \in F_{j}$ with $e \notin m F_{j}+\operatorname{Im} \phi_{j+1}$, codimension $\mathcal{O}\left(\phi_{j}(e)\right) \geq \operatorname{codim} F_{\bullet}+j$, where $\operatorname{codim} F_{\bullet}=\inf \left\{\right.$ codimension $\left.I_{r_{i}}\left(\phi_{i}\right)-i \mid r_{i}=\sum_{j=i}^{s}(-i)^{j-i} \operatorname{rank} F_{j}\right\}$.

The existence of big Cohen-Macaulay modules, due to Hochster ([H2]), played an important role in the proofs of these two theorems.

Bruns and Herzog observed the following as a consequence of the above Theorems.

AMS Subject Classification: Primary 13D02, 13D22, Secondary 13C15, 13D25, 13 H05

Key words and phrases: Projective dimension, grade, order ideal, syzygy.

Typeset by $\mathcal{A} \mathcal{M} \mathcal{S}-\mathrm{T}_{\mathrm{E}} \mathrm{X}$ 
Result (Corollary 9.5.3, $[\mathrm{B}-\mathrm{H}])$. Let $(R, m, k)$ be a regular local ring containing a field and $I \subset m$ be an ideal generated by $x_{1}, \ldots, x_{s}$. Let $K_{\bullet}(\mathbf{x} ; R)$ be the corresponding Koszul complex. Then the natural map $K_{i}(\mathbf{x} ; R) \otimes k \rightarrow \operatorname{Tor}_{i}^{R}(R / I, k)$ is 0 for $i>$ grade $I$.

The question whether the statements of the Theorems and the Result mentioned above are valid in mixed characteristic is very much open. A graded version of Theorem A for mixed characteristic was proved later by Evans and Griffith ([E-G3]). These results induced us to propose the following conjecture ([D1], [D2]).

Order ideal conjecture. Let $(R, m)$ be a local ring and let $M$ be a finitely generated $k$-th syzygy of finite projective dimension and let $x$ be a minimal generator of $M$. Then the order ideal $\mathcal{O}_{M}(x)$ has grade at least $k$.

We say that a finitely generated $\mathrm{R}$-module $\mathrm{N}$ of finite projective dimension over $\mathrm{R}$ satisfies the order ideal conjecture if minimal generators of all its syzygies do so.

In this note we would like to focus our attention to the observation made by Bruns in the above Result. The important question that arises from our study of the Monomial Conjecture, due to Hochster ([H1]), for ideals $I$ of height $d$ in a regular local ring $R$ is the following: given $\mathbf{x}=\left\{x_{1}, \ldots x_{s}\right\}$, a minimal set of generators of $I$, when is $K_{d}(\mathbf{x} ; R) \otimes k \rightarrow$ $\operatorname{Tor}_{d}^{R}(R / I, k)$ the 0-map? In general the answer to the above question is yet to be resolved for any characteristic. We have proved in [D1] that an affirmative answer to the above question for a certain class of ideals implies the Monomial Conjecture. Thus, whether $R$ is equicharacteristic or not, the vanishing of the above map is very significant. We would mainly investigate this question in this note. Our main Result states the following:

Theorem 1.2. Let $(R, m, k)$ be a regular local ring of dimension $n$ and let $I$ be an ideal of height $d$. We have the following:

a) Let $\mathbf{x}=\left\{x_{1}, \ldots, x_{s}\right\}$ denote a minimal set of generators of $I$. If $R / I$ is $S_{2}$, then the natural map $K_{d}(\mathbf{x} ; R) \otimes k \rightarrow \operatorname{Tor}_{d}^{R}(R / I, k)$ is the 0-map.

b) Suppose that $R$ contains a field. Let $\underline{\mathbf{x}}$ (resp. $\mathbf{x}$ ) denote the ideal (resp. R-regular sequence) generated by $x_{1}, \ldots, x_{d}$ in $I$. Let $S=R / \underline{\mathbf{x}}$ and let $\Omega$ denote the canonical module of $R / I$ i.e. $\Omega=\operatorname{Hom}_{S}(R / I, S)=\operatorname{Ext}_{R}^{d}(R / I, R)$. If $\widetilde{\Omega}$ denotes the lift of 
$\Omega$ in $R$ via the natural surjection $R \rightarrow S$, then $K_{d}(\mathbf{x} ; R) \otimes k \rightarrow \operatorname{Tor}_{d}(R / \widetilde{\Omega}, k)$ is the 0-map. Moreover, if $R / I$ is $S_{2}$, then for any set of minimal generators $y_{1}, \ldots, y_{s}$ of $\widetilde{\Omega}, K_{d}\left(y_{1}, \ldots, y_{s} ; R\right) \otimes k \rightarrow \operatorname{Tor}_{d}(R / \widetilde{\Omega}, k)$ is the $0-m a p$.

c) Let $\mathbf{x}$ denote a minimal set of generators of $I$. Then the natural maps $K_{d+i}(\mathbf{x} ; R) \otimes$ $k \rightarrow \operatorname{Tor}_{d+i}(R / I, k)$ are 0-maps for $i>1$.

d) Assume that the order ideal conjecture is valid for regular local rings of dimension $(n-1)$. Let $\mathbf{x}$ denote a minimal set of generators of $I$. If $I \cap\left(m-m^{2}\right) \neq \phi$ or depth $R / I>0$ then the natural maps $K_{d+i}(\mathbf{x} ; R) \otimes k \rightarrow \operatorname{Tor}_{d+i}^{R}(R / I, k)$ are 0-maps for $i \geq 1$. Moreover if $R / I$ is $S_{2}$, then $K_{d+i}(\mathbf{x} ; R) \otimes k \rightarrow \operatorname{Tor}_{d+i}^{R}(R / I, k)$ are 0 -maps for $i \geq 0$

In particular, under the same hypothesis, for any prime ideal $P$ such that $R / P$ is normal, $K_{d+i}(\mathbf{x} ; R) \otimes k \rightarrow \operatorname{Tor}_{d+i}^{R}(R / P, k)$ are 0 -maps for $i \geq 0$.

We would like to mention that in [D1] it was pointed out that the validity of first half of part b) of the above theorem implies the Monomial Conjecture in mixed characteristic.

In the final observation (Prop. 1.3) of this note we prove the existence of ideals $I$ of height $d$ in a regular local ring $(R, m, k)$ for which $K_{i}(\mathbf{x} ; R) \otimes k \rightarrow \operatorname{Tor}_{i}^{R}(R / I, k)$ is non-zero for $i \leq d$; here $\mathbf{x}$ denotes an R-sequence contained in $I$.

\section{SECTION 1}

We intend to prove our theorem in this section.

1.1 Lemma. Let $(R, m)$ be a local ring such that the order ideal conjecture is valid on $R$. Let $I$ be an ideal of $R$ of finite projective dimension over $R$ and let grade $I=d$. Let $x_{1}, \ldots, x_{s}$ be a set of generators of $I$ and let $K_{\bullet}(\mathbf{x} ; R)$ denote the corresponding Koszul complex. Then the natural $K_{i}(\mathbf{x}, R) \otimes k \rightarrow \operatorname{Tor}_{i}^{R}(R / I, k)$ is 0 for $i \geq d+1$.

Proof. The proof is a straightforward application of the validity of the order ideal conjecture as in $(9.5 .3)[\mathrm{B}-\mathrm{H}]$. Let $\left(F_{\bullet}, \phi_{\bullet}\right)$ be a minimal resolution of $R / I$ and let $\psi_{\bullet}$ : $K_{\bullet}(\mathbf{x} ; R) \rightarrow F_{\bullet}$ lift the identity map in $R / I$. If for any minimal generator $e_{j_{1}} \wedge e_{j_{2}} \wedge \cdots \wedge e_{j_{i}}$ of $K_{i}, \psi_{i}\left(e_{j_{1}} \wedge \cdots \wedge e_{j_{i}}\right)=e$ is a minimal generator of $F_{i}$, then by commutativity of the 
diagram constructed via $\psi_{\bullet}, \mathcal{O}\left(\phi_{i}(e)\right)$ has grade $\leq d$. Due to our assumption on order ideals, this can only happen if $i \leq d$.

\subsection{Proof of Theorem $\mathbf{1 . 2}$.}

a) Let $z_{1}, \ldots, z_{d} \in I$ be a regular sequence on $R$ and let $S=R /\left(z_{1}, \ldots, z_{d}\right)$. We will write $\underline{\mathbf{z}}($ resp. $\mathbf{z})$ to denote the ideal (resp. sequence) generated by $z_{1}, \ldots, z_{d}$. Let $\Omega=\operatorname{Hom}_{S}(R / I, S)\left(\simeq \operatorname{Ext}_{R}^{d}(R / I, R)\right)$ denote the canonical module of $R / I$. Let $\widetilde{\Omega}$ denote the lift of $\Omega$ in $R$ via the natural surjection $R \rightarrow S$. Since $R / I$ is $S_{2}, \operatorname{Hom}_{S}(\Omega, \Omega) \simeq R / I$. Hence, by applying $\operatorname{Hom}_{S}(-, S)$ to the short exact sequence

$$
0 \rightarrow \Omega \rightarrow S \rightarrow S / \Omega \rightarrow 0
$$

we obtain $\operatorname{Hom}_{S}(S / \Omega, S)=I / \underline{\mathbf{z}}$ and $\operatorname{Ext}_{S}^{1}(S / \Omega, S)=0$. Since $S / \Omega \simeq R / \widetilde{\Omega}$ and height of $I$ is d, we have

$$
\operatorname{Ext}_{R}^{d}(R / \widetilde{\Omega}, R) \simeq \operatorname{Hom}_{S}(R / \widetilde{\Omega}, S)=\operatorname{Hom}_{S}(S / \Omega, S)=I / \underline{\mathbf{z}}
$$

and

$$
\operatorname{Ext}_{R}^{d+1}(R / \widetilde{\Omega}, R)=\operatorname{Ext}_{S}^{1}(R / \widetilde{\Omega}, S)=\operatorname{Ext}_{S}^{1}(S / \Omega, S)=0 .
$$

Let $F_{\bullet}, P_{\bullet}$ denote the minimal free resolutions of $R / I$ and $I /(\underline{\mathbf{z}})$ respectively and let $K_{\bullet}^{\prime}=K_{\bullet}\left(z_{1}, \ldots, z_{d} ; R\right)$ be the Koszul complex corresponding to the sequence $z_{1}, \ldots, z_{d}$. Let $\psi_{\bullet}: P_{\bullet} \rightarrow K_{\bullet}^{\prime}$ denote a lift of the natural injection $I / \underline{\mathbf{z}} \rightarrow S=R / \underline{\mathbf{z}}$ and let $\phi_{\bullet}: K_{\bullet}^{\prime} \rightarrow$ $F$. denote a lift of the natural surjection $S=R / \underline{\mathbf{z}} \rightarrow R / I$.

Claim. i) $\psi_{d}\left(P_{d}\right)=K_{d}^{\prime}=R$ and ii) $\phi_{d}\left(K_{d}^{\prime}\right) \subset m F_{d}$.

Proof of Claim i). Let $\left(L_{\bullet}, \alpha_{\bullet}\right)$ be a minimal free resolution of $R / \widetilde{\Omega}$ over $R$ and $\theta_{\bullet}: K_{\bullet}^{\prime} \rightarrow$ $L_{\bullet}$ be a lift of the natural surjection $R / \underline{\mathbf{z}} \rightarrow R / \widetilde{\Omega}$. Let $L_{\bullet}^{*}=\operatorname{Hom}_{R}\left(L_{\bullet}, R\right)$. We consider the following commutative diagram

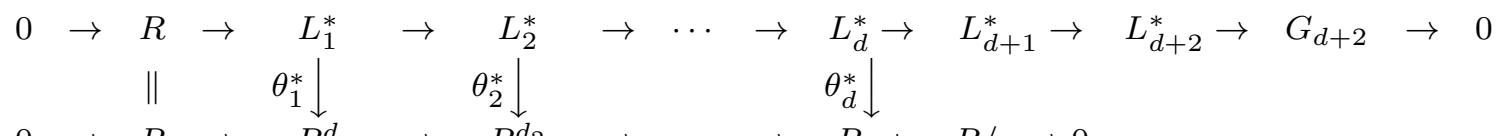

$$
\begin{aligned}
& 0 \rightarrow R \rightarrow R^{d} \rightarrow R^{d_{2}} \rightarrow \cdots \rightarrow R \rightarrow R / \underline{\mathbf{z}} \rightarrow 0
\end{aligned}
$$


Here $G_{d+i}=\operatorname{coker} \alpha_{d+i}^{*}$, for $0 \leq i \leq 2$. Since height $\widetilde{\Omega}$ is $d$, we have $H^{i}\left(L_{\bullet}^{*}\right)=0$ for $i<d$ and $H^{d}\left(L_{\bullet}^{*}\right)=\operatorname{Ext}^{d}(R / \widetilde{\Omega}, R)=I / \underline{\mathbf{z}} \hookrightarrow G_{d}$. Also it follows from (1) that $H^{d+1}\left(L_{\bullet}^{*}\right)=\operatorname{Ext}_{R}^{d+1}(R / \widetilde{\Omega}, R)=0$. Hence $G_{d+1}$ is a submodule of $L_{d+2}^{*}$ and

$$
L_{\bullet}^{\prime}: 0 \rightarrow R \rightarrow L_{1}^{*} \rightarrow \cdots \rightarrow L_{d}^{*} \rightarrow 0
$$

is a minimal free resolution of $G_{d}$. Moreover, the map $G_{d} \rightarrow R / \underline{\mathbf{z}}$, induced by $\theta_{d}^{*}$ in (2), induces the natural inclusion $I / \underline{\mathbf{z}} \hookrightarrow R / \underline{\mathbf{z}}$. Let $\beta_{\bullet}: P_{\bullet} \rightarrow L_{\bullet}^{\prime}$ be a lift of the natural inclusion $I / \underline{\mathbf{z}} \hookrightarrow G_{d}$. We have the following commutative diagram:

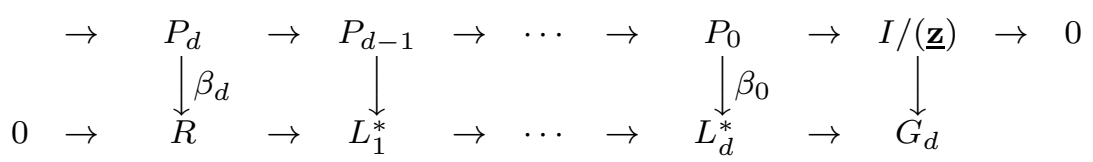

Subclaim. $\beta_{d}\left(P_{d}\right)=R$.

Proof of the Subclaim. If possible, let $\beta_{d}\left(P_{d}\right) \subset m$. Since $\operatorname{coker}\left(I / \underline{\mathbf{z}} \rightarrow G_{d}\right)=\operatorname{Im} \alpha_{d+1}^{*}$, the mapping cone of $\beta_{\bullet}$ gives rise to a free resolution of $G_{d+1}$. Let $\left(Q_{\bullet}, \gamma_{\bullet}\right)$ be a minimal free resolution of $G_{d+1}$ extracted from this mapping cone. Since $\beta_{d}\left(P_{d}\right) \subset m$, the copy of $R=L_{0}{ }^{\prime}$ is a summand of $Q_{d+1}$; we denote this copy by Re. Since height $\widetilde{\Omega}$ is $d$ and $L_{\bullet}$ is a minimal free resolution of $R / \widetilde{\Omega}$, we have

$$
\text { height } \mathcal{O}\left(\gamma_{d+1}(e)\right) \leq d \quad(\text { diagram }(3)) \text {. }
$$

If $R$ is equicharacteristic, this cannot happen due to Theorem $\mathrm{A}$. Let us assume that $R$ is of mixed characteristic $p>0$. Since $G_{d+1}$ is a submodule of $L_{d+2}^{*}, p$ is a non-zero-divisor on $G_{d+1}$. Let $\bar{G}_{d+1}=G_{d+1} \otimes R / p R$ and $\left(\bar{Q}_{\bullet}, \bar{\gamma}_{\bullet}\right)=\left(Q \bullet \otimes R / p R, \gamma_{\bullet} \otimes R / p R\right)$. Then $\left(\bar{Q}_{\bullet}, \bar{\gamma}_{\bullet}\right)$ is a minimal free resolution of $\bar{G}_{d+1}$ over $\bar{R}(=R / p R)$. Since $\bar{R}$ is an equicharacteristic local ring (characteristic $p>0$ ), by Theorem A, height $\mathcal{O}\left(\gamma_{d+1}(\bar{e})\right)$ must be $\geq d+1$. This contradicts (4) and hence $\beta_{d}\left(P_{d}\right)=R$.

Since $\psi_{\bullet}$ can be constructed as the composite of $\theta_{\bullet}^{*}$ and $\beta_{\bullet}$, the above observation implies claim i) i.e. $\psi_{d}\left(P_{d}\right)=K_{d}^{\prime}=R$ via combination of diagrams (2) and (3). 
Proof of claim ii). Since $\psi_{d}\left(P_{d}\right)=R$, in the minimal free resolution $F_{\bullet}$ of $R / I$ extracted from the mapping cone of $\psi_{\bullet}$, the copy of $K_{d}^{\prime}=R$ does not survive as a minimal generator of $F_{d}$ i.e. $\phi_{d}\left(K_{d}^{\prime}\right) \subset m F_{d}$, where $\phi_{\bullet}$ is extracted from the natural inclusion of $K_{\bullet}^{\prime}$ into the mapping cone of $\psi$.

Thus both parts of our claim are proved. Since height $I$ is $d$ and $R$ is regular local, grade $I$ is also $d$. We can find a minimal set of generators $x_{1}, \ldots, x_{s}$ of $I$ such that any subset $x_{i_{1}}, \ldots, x_{i_{d}}, 1 \leq i_{1}<i_{2}<\cdots<i_{d} \leq s$, form an $R$-sequence. Let $K_{\bullet}=K \bullet\left(x_{1}, \ldots, x_{s} ; R\right)$ denote the Koszul complex corresponding to $x_{1}, \ldots, x_{s}$. Let $F_{\bullet}$ be a minimal free resolution of $R / I$ over $R$ and let $\phi_{\bullet}: K_{\bullet} \rightarrow F_{\bullet}$ denote a lift of the identity map on $R / I$. By part ii) of our claim, $\phi_{d}\left(R e_{i_{1}}, \wedge \cdots \wedge e_{i_{d}}\right) \subset m F_{d}, \forall d$-tuple $\left(i_{1}, \ldots, i_{d}\right)$ such that $1 \leq i_{1}<i_{2}<$ $\cdots<i_{d} \leq s$. Hence $K_{d}(\mathbf{x} ; R) \otimes R / m \rightarrow F_{d} \otimes R / m$ is the 0-map.

b) Let $S=R / \underline{\mathbf{x}}$ and let $\Omega=\operatorname{Hom}_{S}(R / I, S)=\operatorname{Ext}_{R}^{d}(R / I, R)$. Let $\left(P_{\bullet}, \alpha_{\bullet}\right)$ be a minimal free resolution of $R / I$ over $R$; let $P_{\bullet}^{*}=\operatorname{Hom}_{R}(P, R)$. We consider the following complex truncated from $P_{\bullet}^{*}$ :

$$
0 \rightarrow R \rightarrow P_{1}^{*} \rightarrow \cdots \rightarrow P_{d}^{*} \rightarrow P_{d+1}^{*} \rightarrow G_{d+1} \rightarrow 0
$$

where $G_{d+1}=\operatorname{coker} \alpha_{d+1}^{*}$ and $G_{d}=\operatorname{coker} \alpha_{d}^{*}$. We have $H^{i}\left(P_{\bullet}^{*}\right)=0$ for $i<d$ and $H^{d}\left(P_{\bullet}^{*}\right)=\Omega$. Let $P_{\bullet}^{\prime}: 0 \rightarrow R \rightarrow P_{1}^{*} \rightarrow \cdots \rightarrow P_{d}^{*} \rightarrow 0$ denote the minimal free resolution of $G_{d}$ obtained from (1). Let $F_{\bullet}$ be a minimal free resolution of $\Omega$ over $R$ and let $\phi_{\bullet}: F_{\bullet} \rightarrow P_{\bullet}^{\prime}$ lift the natural inclusion $\Omega \hookrightarrow G_{d}$. $R$ being equicharacteristic, Theorem $\mathrm{A}$ is valid in $R$. Since the mapping cone of $\phi_{\bullet}$ gives rise to a free resolution of $G_{d+1}$, arguing as in the proof of part a), it follows that $\phi_{d}\left(F_{d}\right)=R$. Let $\theta_{\bullet}: K_{\bullet}(\mathbf{x} ; R) \rightarrow P_{\bullet}$ denote a lift of the natural surjection $R / \underline{\mathbf{x}} \rightarrow R / I$ and let $\theta_{\bullet}^{*}: P_{\bullet}^{\prime} \rightarrow K_{\bullet}(\mathbf{x} ; R)$ denote the dual map that lifts the map $G_{d} \rightarrow R / \underline{\mathbf{x}}$ induced by $\theta_{d}^{*}$. Then $\theta_{\bullet}^{*} \cdot \phi_{\bullet}: F_{\bullet} \rightarrow K_{\bullet}(\mathbf{x} ; R)$ lifts the natural injection $\Omega \hookrightarrow R / \underline{\mathbf{x}}$ (composition of $\Omega \hookrightarrow G_{d} \rightarrow R / \underline{\mathbf{x}}$ ) and $\theta_{0}^{*} \phi_{d}\left(F_{d}\right)=R$. Let $L$. be a minimal free resolution of $R / \widetilde{\Omega}$ extracted from the mapping cone of $\theta_{\bullet}^{*} \cdot \phi_{\bullet}$ and $\psi_{\bullet}: K_{\bullet}(\mathbf{x} ; R) \rightarrow L_{\bullet}$ be a lift of the natural surjection $R / \underline{\mathbf{x}} \rightarrow R / \widetilde{\Omega}$ induced by the inclusion map of $K_{\bullet}(\mathbf{x} ; R)$ into the mapping cone of $\theta_{\bullet}^{*} . \phi_{\bullet}$. Again arguing as in the proof of part a) we obtain $\psi_{d}\left(K_{d}(\mathbf{x} ; R)=R\right) \subset m L_{d}$. Hence the natural map $K_{d}(\mathbf{x} ; R) \otimes k \rightarrow \operatorname{Tor}_{d}^{R}(R / \widetilde{\Omega}, k)$ is the 
0-map.

Now assume $R / I$ is $S_{2}$. Then $\operatorname{Ext}_{R}^{d+1}(R / \widetilde{\Omega}, R)=0\left((1)\right.$ in part a)). Let $\mathbf{y}=\left\{y_{1}, \ldots, y_{d}\right\}$ be a part of a minimal set of generators of $\widetilde{\Omega}$ such that they form an $R$-sequence. Let $T=R /\left(y_{1}, \ldots, y_{d}\right)$, and $\widetilde{\Omega} /\left(y_{1}, \ldots, y_{d}\right)=\omega ;$ then $T / \omega=R / \widetilde{\Omega}$ and $\operatorname{Ext}_{T}^{1}(T / \omega, T)=$ $\operatorname{Ext}_{R}^{d+1}(R / \widetilde{\Omega}, R)=0$.

Let $J=\operatorname{Hom}_{T}(T / \omega, T)=\operatorname{Ext}_{R}^{d}(R / \widetilde{\Omega}, R)$. We consider the short exact sequence

$$
0 \rightarrow \omega \rightarrow T \rightarrow T / \omega \rightarrow 0
$$

Applying $\operatorname{Hom}_{T}(\ldots, T)$ to this sequence, we obtain

$$
0 \rightarrow J \rightarrow T \rightarrow \operatorname{Hom}_{T}(\omega, T) \rightarrow 0
$$

Hence $T / J \simeq \omega^{*}=\operatorname{Hom}_{T}(\omega, T)$. Since $T / \omega=R / \widetilde{\Omega}$ is $S_{1}, \omega$ is $S_{2}$ and hence $\operatorname{Hom}_{T}(T / J, T)=$ $\omega$.

Let $\widetilde{J}$ be a lift of $J$ in $R$ via the natural surjection $R \rightarrow T$. Let $K_{\bullet}(\mathbf{y} ; R)$ denote the Koszul complex corresponding to $y_{1}, \ldots, y_{d}$. Let $B$ • be a minimal free $R$-resolution of $\omega$ and let $L_{\bullet}$ be a minimal free resolution of $T / \omega=R / \widetilde{\Omega}$ over $R$. Then, arguing as in part a), starting with a minimal free resolution of $R / \widetilde{J}$ over $R$ we can show that the natural map $K_{d}(\mathbf{y} ; R) \otimes k \rightarrow \operatorname{Tor}_{d}^{R}(R / \widetilde{\Omega}, k)$ is the 0 -map. Now considering a minimal set of generators $y_{1}, \ldots, y_{s}$ of $\widetilde{\Omega}$ such for any $d$-tuples $\left(i_{1}, \ldots, i_{d}\right), 1 \leq i_{1}<i_{2}<\cdots<i_{d} \leq s, y_{i_{1}}, \ldots, y_{i_{d}}$ from an $R$-sequence, it follows that the natural map $K_{d}\left(y_{1}, \ldots, y_{s} ; R\right) \otimes k \rightarrow \operatorname{Tor}_{d}^{R}(R / \widetilde{\Omega}, k)$ is the 0-map.

c) This follows from the facts that for a minimal free resolution $F_{\bullet}$ of a finitely generated module $M$ over $R$, the $(d+1)$ th syzygy $\operatorname{Syz}_{R}^{d+1}(M)=\operatorname{Syz}_{R}^{d}\left(S_{1}\right)$ where $S_{1}=\operatorname{Syz}_{R}^{1}(M)$ and the mixed characteristic $p$ is a non-zero-divisor on $S_{1}$. Let $\bar{R}=R / p R$. Then $\bar{F} \bullet=$ $F_{\bullet}(+1) \otimes \bar{R}$ is a minimal free resolution of $\bar{S}_{1}=S_{1} \otimes \bar{R}$ over $\bar{R}$. Since $\bar{R}$ is equicharacteristic, Theorem $\mathrm{A}$ is valid on $\bar{R}$ and hence our assertion follows.

d) If $\mathrm{w} \epsilon\left(m-m^{2}\right)$ is a non-zero-divisor on $R / I$, the result follows by similar arguments as in the proof of part c) above.

If $\mathrm{w} \epsilon\left(m-m^{2}\right) \cap I$, the result follows from the proof of Lemma (1.1) and the following theorem which is a part of theorem 2.5 in [D2]. 
Theorem (Theorem 2.5, [D2]). Let $(R, m)$ be a regular local ring and let $M$ be a module of finite projective dimension over $R$. Assume that the order ideal conjecture is valid for regular local rings of dimension ( $n$-1). If ann $n_{R} M \cap\left(m-m^{2}\right) \neq \phi$ or depth $h_{R} M>0$, then $M$ satisfies the order ideal conjecture.

For a proof we refer the reader to theorem 2.5 in ([D2]).

The last part of the statement in d) now follows from a) and the above theorem.

1.3. In the final proposition we prove the existence of almost complete intersection ideals I of height d for which $K_{i}(\mathbf{x} ; R) \otimes k \rightarrow \operatorname{Tor}_{i}^{R}(R / I, k)$ is non-zero for $0 \leq i \leq d$.

Proposition. Let $(R, m, k)$ be a regular local ring and let $P$ be a prime ideal of height $d$. Then there exists a family of almost complete intersection ideals $\left\{I_{t}\right\}_{t \in N} \subset P$ such that $K_{i}(\mathbf{x} ; R) \otimes k \rightarrow \operatorname{Tor}_{i}^{R}\left(R / I_{t}, k\right)$ is injective for $i \geq 0$ and $t>1$; here $\mathbf{x}$ denotes a part of a minimal set of generator $x_{1}, \ldots, x_{d}$ of $I_{t}$ for all $t$.

Proof. We choose $x_{1}, \ldots, x_{d} \in P$ such that $x_{1}, \ldots, x_{d}$ form an R-sequence and $P R_{P}=$ $\underline{\mathbf{x}} R_{P} ; \underline{\mathbf{x}}=$ the ideal generator by $x_{1}, \ldots, x_{d}$. We have a primary decomposition of $\underline{\mathbf{x}}$ as follows:

$$
\underline{\mathbf{x}}=P \cap q_{2} \cap \cdots \cap q_{r}
$$

where $q_{i}$ is $P_{i}$-primary, $2 \leq i \leq r$. Let $\Omega$ denote the canonical module for $R / P$; then $\Omega=\operatorname{Ext}_{R}^{d}(R / P, R)=\operatorname{Hom}_{R}(R / P, R / \underline{\mathbf{x}})=q_{2} \cap \cdots \cap q_{r} / \underline{\mathbf{x}}$. Let $\lambda \in P-\cup P_{i}$. It can be easily checked that $\Omega=\operatorname{Hom}_{R}\left(R /\left(\underline{\mathbf{x}}, \lambda^{t}\right), R / \underline{\mathbf{x}}\right)$ for $t>0$. Let $\Omega^{\prime}$ denote the lift of $\Omega$ in $\mathrm{R}$ via the natural surjection $R \rightarrow R / \underline{\mathbf{x}}$. Then $\left(\underline{\mathbf{x}}+\lambda^{t} R\right) / \lambda^{t} R \simeq \underline{\mathbf{x}} / \underline{\mathbf{x}} \cap \lambda^{t} R=\underline{\mathbf{x}} / \lambda^{t} \Omega^{\prime}$ for $t>0$. Consider the injection $i_{t}: \Omega^{\prime} \rightarrow \underline{\mathbf{x}}$ defined by $i_{t}(\mu)=\lambda^{t} \mu$. Let $F_{\bullet}$ denote a minimal free resolution of $\Omega^{\prime}, K_{\bullet}=K_{\bullet}(\mathbf{x} ; R)(+1)$ (i.e. $\left.K_{i}=K_{i+1}(\mathbf{x} ; R)\right)$ denote the minimal free resolution of $\underline{\mathbf{x}}$ and let $\Phi_{\bullet}: F_{\bullet} \rightarrow K_{\bullet}$ denote a lift of $i_{t}$. Since $\lambda \bullet i_{t}=i_{t+1}, \lambda . \Phi_{\bullet}$ is a lift of $i_{t+1}$. Let us assume that $t>1$. Then $\operatorname{im} \Phi_{j} \subset m K_{j}$ for $j \geq 0$. Hence $L_{\bullet}$, the mapping cone of $\Phi_{\bullet}$, is a minimal free resolution of $\underline{\mathbf{x}} / \lambda^{t} \Omega^{\prime}$. Hence, via the natural injection of $K \bullet$ into $L_{\bullet}, K_{\bullet}$ imbeds into $L_{\bullet}$ i.e. $K_{j}$ is a free summand of $L_{j}$ for $j \leq d-1$. Let $K_{\bullet}\left(\lambda^{t} ; R\right)$ be the Koszul complex corresponding to $\lambda^{t}$ over R. Let $\Psi_{\bullet}: L_{\bullet} \rightarrow K_{\bullet}\left(\lambda^{t} ; R\right)$ lift the inclusion 
map : $\underline{\mathbf{x}} / \lambda^{t} \Omega^{\prime} \hookrightarrow R / \lambda^{t} R$. Then the mapping cone $P_{\bullet}$ of $\Psi_{\bullet}$ is a minimal free resolution of $R /\left(\underline{\mathbf{x}}, \lambda^{t}\right)$ and it is easy to check that $K_{\bullet}(\mathbf{x} ; R)$ imbeds into $P \bullet$ i.e. $K_{i}(\underline{\mathbf{x}} ; R)$ is a free summand of $P_{i}$ for $i \geq 0$. Hence $K_{i}(\underline{\mathbf{x}} ; R) \otimes k \rightarrow \operatorname{Tor}_{i}^{R}\left(R /\left(\underline{\mathbf{x}}, \lambda^{t}\right), k\right)$ is injective for $i \geq 0$ and our proof is complete.

\section{REFERENCES}

[B-H] W. Bruns and J. Herzog, Cohen-Macaulay Rings, Cambridge Studies in Advanced Mathematics 39, Cambridge University Press, 1993.

[D1] S. P. Dutta, The Monomial Conjecture and Order Ideals, Journal of Algebra 22 (2013), 85 - 103.

[D2] - On Modules of Finite Projective Dimension, Preprint.

[E-G1] E. G. Evans and P. Griffith, The Syzygy Problem, Annals of Mathematics 114 (1981), 323-333.

[E-G2] , Order Ideals, in M. Hochster, J. D. Sally, and C. Huneke (eds.), Commutative Algebra, Math. Sc. Res. Inst. Publ. 15, Springer (1989), 213-225.

[E-G3] _ A Graded Syzygy Theorem in Mixed Characteristic, Math. Research Letters 8 (2001), 605-611.

[H1] M. Hochster, Contracted Ideals from Integral Extensions of Regular rings, Nagoya Math. J. 51 (1973), 25-43.

[H2] Topics in the Homological Theory of Modules over Commutative rings, C.B.M.S. Regional Conference Series in Math, \#24, Amer. Math. Soc., Providence, RI 1975.

UNIVERSITY OF ILLINOIS

Department of Mathematics

1409 West Green Street

URBANA, IL 61801

U.S.A.

E-mail: dutta@math.uiuc.edu 